\title{
Annular Fast Electron Transport in Silicon Arising from Low-Temperature Resistivity
}

\author{
D. A. MacLellan, ${ }^{1}$ D. C. Carroll,,${ }^{1}$ R. J. Gray, ${ }^{1}$ N. Booth, ${ }^{2}$ M. Burza, ${ }^{3}$ M. P. Desjarlais, ${ }^{4}$ F. Du, ${ }^{5}$ \\ B. Gonzalez-Izquierdo, ${ }^{1}$ D. Neely, ${ }^{2}$ H. W. Powell, ${ }^{1}$ A. P. L. Robinson, ${ }^{2}$ D. R. Rusby, ${ }^{1,2}$ \\ G. G. Scott, ${ }^{1,2}$ X. H. Yuan, ${ }^{6}$ C.-G. Wahlström, ${ }^{3}$ and P. McKenna ${ }^{1, *}$ \\ ${ }^{1}$ Department of Physics, SUPA, University of Strathclyde, Glasgow G4 ONG, United Kingdom \\ ${ }^{2}$ Central Laser Facility, STFC Rutherford Appleton Laboratory, Oxfordshire OX11 OQX, United Kingdom \\ ${ }^{3}$ Department of Physics, Lund University, Post Office Box 118, S-22100 Lund, Sweden \\ ${ }^{4}$ Sandia National Laboratories, Post Office Box 5800, Albuquerque, New Mexico 87185, USA \\ ${ }^{5}$ Beijing National Laboratory of Condensed Matter Physics, Institute of Physics, Chinese Academy of Sciences, Beijing 100190, China \\ ${ }^{6}$ Department of Physics and Key Laboratory for Laser Plasmas (Ministry of Education), \\ Shanghai Jiao Tong University, Shanghai 200240, China \\ (Received 3 April 2013; published 28 August 2013)
}

Fast electron transport in $\mathrm{Si}$, driven by ultraintense laser pulses, is investigated experimentally and via 3D hybrid particle-in-cell simulations. A transition from a Gaussian-like to an annular fast electron beam profile is demonstrated and explained by resistively generated magnetic fields. The results highlight the potential to completely transform the beam transport pattern by tailoring the resistivity-temperature profile at temperatures as low as a few $\mathrm{eV}$.

Understanding the transport of high currents of fast $(\mathrm{MeV})$ electrons in dense targets irradiated by ultraintense laser pulses is not only of fundamental interest but is also important for many applications such as the fast-ignition approach to inertial confinement fusion (ICF) [1] and the development of laser-driven ion sources [2]. Techniques involving target engineering $[3,4]$ and multiple laser pulses [5] have been explored to control key fast electron beam properties, such as divergence. An ability to completely change the transport pattern could have radical implications for applications such as fast-ignition ICF and for generating tailored ion beams for use in medicine and industry. In particular, the use of annular beams of fast electrons and protons is predicted to significantly lower the energy requirements of the drive laser pulse for fast-ignition ICF [6,7].

Previous studies have shown that fast electron propagation in solids is subject to a variety of phenomena, many of which depend strongly on target resistivity, including electric-field inhibition [8], resistive instabilities [9], and self-generated resistive magnetic fields [10]. Although the overall importance of resistivity is widely acknowledged, its influence at temperatures below $\sim 20 \mathrm{eV}$ has been largely unexplored. This is because many experimental studies use relatively thin targets (tens of microns) for which the majority of the transport region is heated to higher temperatures, at which resistivity is adequately described by the Spitzer formula [11]. For fast electron transport in thicker targets, for which less heating of the bulk target occurs, the resistivity evolution at lower temperatures must be considered. Previously, we showed that the onset of resistive instabilities in the fast electron beam transport depends strongly on the resistivity in the tens of
$\mathrm{eV}$ temperature regime, which in turn is defined by the target lattice structure [12].

In this Letter, we demonstrate for the first time that completely new types of fast electron transport patterns can be generated by understanding and controlling the target resistivity evolution at temperatures as low as a few eV. We measure a signature of an annular beam profile in silicon and, via comparison with results from 3D hybrid particle-in-cell (PIC) modeling, show that this transport pattern originates from a dip in resistivity at $\sim 3 \mathrm{eV}$. The results demonstrate the importance of properly accounting for low-temperature resistivity in investigation of fast electron transport.

Fast electron transport simulations in silicon were performed using three different resistivity-temperature models, shown in Fig. 1. The Spitzer model [11] accounts for ionization and collisions of plasma particles but is only applicable for fully ionized, nondegenerate plasmas (which typically only occurs within the first few tens of microns depth in an intense laser-solid interaction). The Lee-More model [13] is a wide-range density-temperature resistivity calculation, based upon the Thomas-Fermi ionization model. It does not include atomic structure effects and is therefore of limited validity for insulator-conductor (and semiconductor-conductor) transitions. A more accurate determination of the low-temperature resistivity of silicon is achieved using ab initio quantum molecular dynamics (QMD) simulations, based on density functional theory [14]. The Vienna ab initio simulation program (VASP), a plane-wave density functional code $[15,16]$, was used. To produce the profile shown in Fig. 1, the silicon atomic configurations were obtained from $300 \mathrm{~K}$ simulations. The electronic temperature was then varied from 1 to 


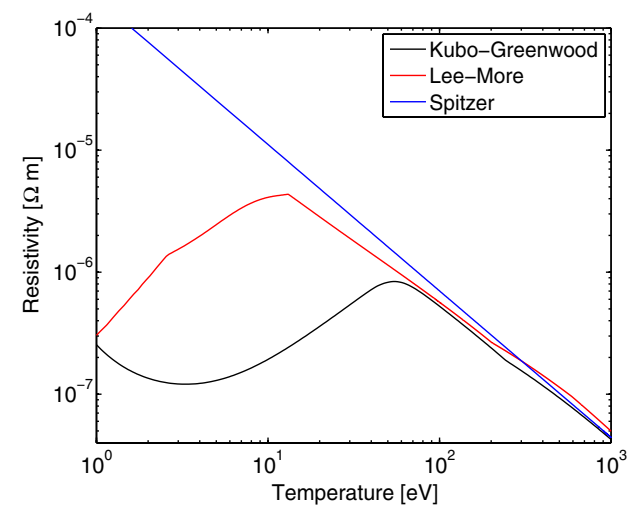

FIG. 1 (color online). Theoretical calculations of the resistivity of silicon as a function of temperature: The black line represents $a b$ initio QMD calculations coupled with the Kubo-Greenwood equation, the red line the Lee-More model, and the blue line the Spitzer model.

$20 \mathrm{eV}$ in subsequent static Kubo-Greenwood conductivity calculations [15] using the previously sampled atomic configurations. As shown in Fig. 1, the three resistivity profiles differ greatly at temperatures below $50 \mathrm{eV}$.

The minimum at $\sim 3.5 \mathrm{eV}$ in the QMD KuboGreenwood profile corresponds to excitation of electrons above the band gap of $1.12 \mathrm{eV}$, giving rise to more charge carriers in the conduction band and an overall reduction in the resistivity. Thereafter, the resistivity rises to peak at $\sim 50 \mathrm{eV}$, which represents a transition from electronphonon scattering to electron-ion collisions and a resulting minimum in the electron mean free path. Beyond $\sim 50 \mathrm{eV}$, ionization dominates and scattering cross sections decrease with increasing mean electron momentum, giving rise to the Spitzer resistivity.

A series of simulations of fast electron transport in silicon were performed using the three resistivitytemperature models incorporated into the 3D hybrid code ZEPHYROS $[3,17]$ to investigate the effects of lowtemperature resistivity. ZEPHYROS treats the fast electrons kinetically and the background using a hybrid description, as in the code outlined by Davies et al. [18,19]. A $200 \mu \mathrm{m} \times 400 \mu \mathrm{m} \times 400 \mu \mathrm{m}$ simulation grid was used (target thickness equal to $200 \mu \mathrm{m}$ ), with a cell resolution of $\Delta X=\Delta Y=\Delta Z=1 \mu \mathrm{m}$. The laser-to-fast electron energy conversion factor was set to 0.3 , with pulse duration of $1 \mathrm{ps}$. A relativistic Maxwellian distribution of electron energies was used with mean temperature (assuming ponderomotive scaling [20]) equal to 1.3 and $6.2 \mathrm{MeV}$, for peak laser intensities equal to $5 \times 10^{19}$ and $5 \times$ $10^{20} \mathrm{~W} \mathrm{~cm}^{-2}$, respectively. The electron injection halfangle was set to $50^{\circ}$ [21]. In all cases, the initial target temperature was set equal to $1 \mathrm{eV}$, except for simulations involving the Spitzer model, as discussed below. The target resistivity evolves with temperature in accordance with the selected model (Fig. 1). Example simulation results are shown in Fig. 2.
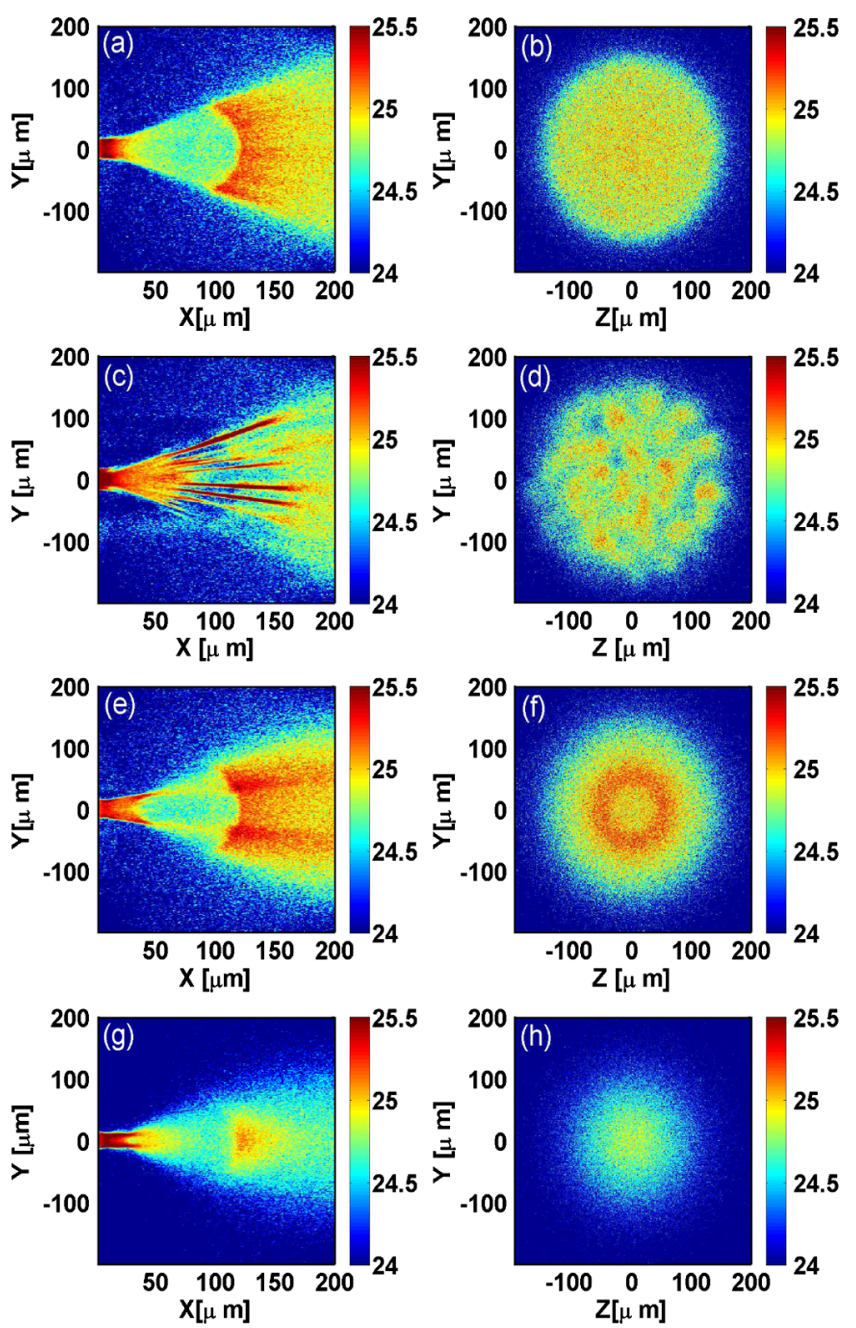

FIG. 2 (color online). Hybrid PIC simulation results showing $\log _{10}$ fast electron density maps $\left(\mathrm{m}^{-3}\right)$ in the $[X-Y]$ midplane and rear-surface $[Y-Z]$ plane, $1.4 \mathrm{ps}$ after laser irradiation. (a),(b) Spitzer, (c),(d) Lee-More, and (e),(f) QMD KuboGreenwood models, all for peak intensity equal to $5 \times 10^{20} \mathrm{~W} \mathrm{~cm}^{-2}$. (g),(h) Corresponding simulation result for the QMD Kubo-Greenwood resistivity-temperature calculations at $5 \times 10^{19} \mathrm{~W} \mathrm{~cm}^{-2}$.

The electron population is injected at $[X, Y, Z]=$ $[0,0,0]$ and propagates in the $X$ direction. Snapshots of the fast electron density in the $[X, Y]$ midplane and $[Y, Z]$ rear plane are shown for four given cases. Figures 2(a)-2(f) correspond to the use of the Spitzer, Lee-More, and QMD Kubo-Greenwood models, respectively, for electron beam parameters corresponding to peak laser intensity equal to $5 \times 10^{20} \mathrm{~W} \mathrm{~cm}^{-2}$. Figures $2(\mathrm{~g})$ and $2(\mathrm{~h})$ are the corresponding result for the QMD Kubo-Greenwood case at a lower peak intensity of $5 \times 10^{19} \mathrm{~W} \mathrm{~cm}^{-2}$.

Three distinctly different fast electron transport patterns are obtained. Using the Spitzer model, with an initial target temperature of $100 \mathrm{eV}$ (noting that all three models converge to the same resistivity at high temperatures), results in smooth electron transport and a relatively uniform 
density distribution at the target rear [Figs. 2(a) and 2(b)]. The Spitzer model is not valid at low temperatures, and test simulations at a starting temperature of $1 \mathrm{eV}$ result in an unphysical high magnetic field strength in the region of the electron source, which prevents beam propagation. As shown in Figs. 2(c) and 2(d), the Lee-More model correction to the Spitzer resistivity at low-temperature results in strongly filamented beam transport, leading to a highly structured rear-surface density distribution. In contrast, the QMD Kubo-Greenwood approach produces a distinctly different transport profile, resulting in an annular fast electron density distribution, as shown in Figs. 2(e) and 2(f). The transport pattern is sensitive to the laser intensity. Figures 2(g) and 2(h) show corresponding results for the $5 \times 10^{19} \mathrm{~W} \mathrm{~cm}^{-2}$ case, for which smooth, uniform beam transport occurs. We note that there is evidence of the seeding of a beam hollowing effect at $X=40 \mu \mathrm{m}$ at both intensities, but only in the higher intensity case does this develop into an annular beam profile.

To test these predictions, we performed an experiment using the Vulcan laser at the Rutherford Appleton Laboratory, UK. The laser delivered $1.055 \mu \mathrm{m}$ wavelength light in $(0.8 \pm 0.2)$ ps duration [full width at half maximum (FWHM)] pulses, with a maximum energy (on target) of $300 \mathrm{~J}$. The $p$-polarized pulses were focused using an $f / 3$ off-axis parabolic mirror, at an incident angle of $33^{\circ}$, to a $4.5 \mu \mathrm{m}$ diameter (FWHM) spot. This produced a calculated peak intensity of $6.8 \times 10^{20} \mathrm{~W} \mathrm{~cm}^{-2}$. The targets were $300-\mu \mathrm{m}$-thick $3 \mathrm{~mm} \times 3 \mathrm{~mm}$ silicon. We choose thick targets to avoid fast electron refluxing, which can strongly affect magnetic field generation and thus electron transport physics in thin targets [22]. The transport patterns were diagnosed by measuring the spatial-dose distribution of the beam of protons accelerated by the sheath field established by the arrival of the fast electrons at the target rear surface $[12,23,24]$. The 2D fast electron density profile is mapped into the sheath field and thereby the spatialintensity distribution of the resulting proton beam. The $2 \mathrm{D}$ dose distribution is measured at discrete proton energies using a stack of dosimetry film (radiochromic film) positioned $6 \mathrm{~cm}$ from the rear surface of the target and centered on the target normal. Figures 3(a)-3(c) show representative measurements (all at an example energy of $7.3 \mathrm{MeV}$ - the same features are measured at all energies, i.e., throughout the radiochromic film stack), as a function of peak laser intensity between $6 \times 10^{19}$ and $6.8 \times 10^{20} \mathrm{~W} \mathrm{~cm}^{-2}$ (respective laser pulse energies of 26 and $300 \mathrm{~J}$ ) for otherwise identical laser pulse and target parameters.

The smooth proton beam measured at $6 \times 10^{19} \mathrm{~W} \mathrm{~cm}^{-2}$ [Fig. 3(a)] is indicative of smooth electron transport. The slight intensity enhancement in the beam center may indicate the onset of structure. As the peak laser intensity is increased to $4 \times 10^{20} \mathrm{~W} \mathrm{~cm}^{-2}$ [Fig. 3(b)], the overall beam size grows and several ringlike structures start to form within it, close to the center. At the highest intensity
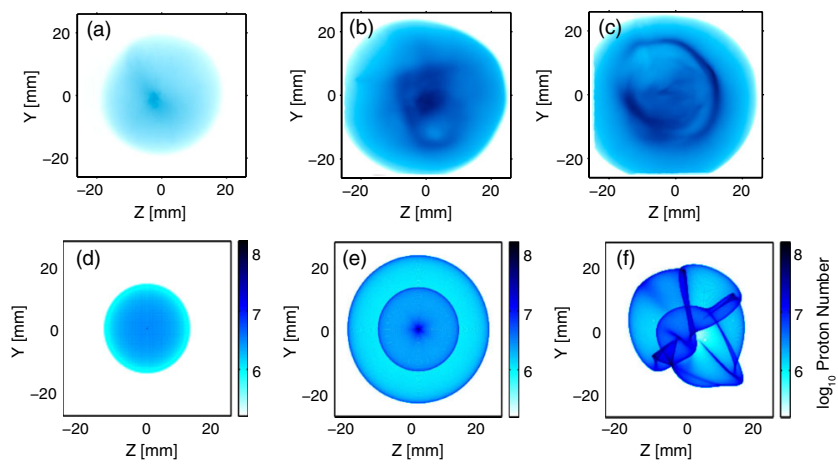

FIG. 3 (color online). (a)-(c) Representative example measured proton spatial-intensity distributions at $7.3 \mathrm{MeV}$ for peak laser intensity equal to: (a) $6 \times 10^{19} \mathrm{~W} \mathrm{~cm}^{-2}$, (b) $4 \times 10^{20} \mathrm{~W} \mathrm{~cm}^{-2}$, and (c) $6.8 \times 10^{20} \mathrm{~W} \mathrm{~cm}^{-2}$. (d)-(f) Analytical model calculations of the proton spatial distribution resulting from the rear-surface fast electron density distributions shown in (d) Fig. 2(h) $\left(5 \times 10^{19} \mathrm{~W} \mathrm{~cm}^{-2}\right)$, (e) Fig. 2(f) $\left(5 \times 10^{20} \mathrm{~W} \mathrm{~cm}^{-2}\right)$, and (f) Fig. 2(d) $\left(5 \times 10^{20} \mathrm{~W} \mathrm{~cm}^{-2}\right)$.

accessible [Fig. 3(c)], a single clear annular profile is measured within the beam. From this, we conclude that the fast electron beam transport pattern is sensitive to the peak laser intensity and that a ringlike pattern emerges at the highest intensity accessible, in agreement with simulation results with the QMD Kubo-Greenwood model, shown in Figs. 2(e)-2(h).

To enable a more direct comparison between the proton beam measurements and the fast electron transport simulation results, an analytical model was developed to compute the evolution of the 2D sheath field and the resulting proton production and projection [24]. The 2D electricfield distribution is calculated using the rear-surface fast electron density distribution results from the ZEPHYROS simulations. The spreading of the fast electrons on the target surface and the resulting evolution of the sheath field are calculated. The protons are produced assuming field ionization of hydrogen and the local gradients in the proton front are used to calculate their projection onto the detector plane.

Figures 3(d) and 3(e) show the calculated proton beam spatial-intensity distributions resulting from the QMD Kubo-Greenwood model fast electron density maps at the two intensities, i.e., Figs. 2(h) and 2(f), respectively. Excellent qualitative agreement is found between Figs. 3(a) and 3(d) for the lower intensity, for which smooth transport results in a uniform proton beam, and Figs. 3(c) and 3(e) at the higher intensity, for which the annular fast electron beam observed in the simulations results in a ringlike structure in the proton beam. These results contrast sharply with the distinctly different proton beam profiles, without annular features, which are predicted using the fast electron density distributions simulated with the other resistivity-temperature profiles. A smooth, circular proton beam intensity distribution is 
calculated using the Spitzer fast electron density distribution [Fig. 2(b)], and the filamented fast electron beam profile for the Lee-More model [Fig. 2(d)] results in an irregular proton beam with strong cusplike structures within it, as shown in Fig. 3(f) (similar to those observed previously, e.g., Ref. [12]).

To investigate why low-temperature resistivity plays such an important role, we consider how evolving temperature, and therefore resistivity, gradients subsequently lead to magnetic field patterns which strongly influence electron propagation. The growth rate of the selfgenerated, resistive magnetic field is described by [25]

$$
\frac{\partial \mathbf{B}}{\partial t}=\eta \boldsymbol{\nabla} \times \mathbf{J}_{f}+\nabla \eta \times \mathbf{J}_{f}
$$

where $\mathbf{J}_{f}$ is the fast electron current density and $\eta$ is the target resistivity. The first term on the right-hand side of Eq. (1) produces a magnetic field which forces electrons towards regions of highest current density. This results in a pinching action on the beam [10]. The second term arises from resistivity gradients in the target and generates a field that pushes electrons towards higher resistivity regions [25]. As described by Davies [25], the latter effect can lead to a hollowing of the fast electron beam. In that scenario, as the beam density is initially centrally peaked (typically a Gaussian profile), the highest collisional return current is drawn, and therefore the largest degree of target heating occurs on axis. As the beam propagates into a region of the target in which resistivity decreases with increasing temperature (the Spitzer regime is considered by Davies [25]), the target becomes less resistive on axis than at larger radii, which could lead to beam hollowing if the field due to the second term in Eq. (1) exceeds the collimating effect of the field due to the first.

In the case of silicon, we find that it is the dip in the resistivity profile (Fig. 1) at low temperatures, centered at $\sim 3.5 \mathrm{eV}$, combined with the action of the second term in Eq. (1), which is key to inducing the observed annular structure. To illustrate this, Fig. 4 shows example ZEPHYROS simulation results of silicon target heating and resulting resistivity and magnetic fields. As shown in the 2D temperature map $\left(5 \times 10^{20} \mathrm{~W} \mathrm{~cm}^{-2}\right.$ case $)$ [Fig. 4(a)] and in the example transverse temperature profiles [Fig. 4(b)], the target is heated to relatively low temperatures, in the eV range (depending on laser drive intensity) at a depth of $\sim 50 \mu \mathrm{m}$. Initially, the highest temperature is on axis for the Gaussian profile beam. At the edge of the beam, where the temperature drops to $\sim 3.5 \mathrm{eV}$, the resistivity gradient changes sign, as shown in Fig. 4(d), due to the dip in the resistivity-temperature profile (dashed lines are shown in Fig. 4 to illustrate this correlation for both laser intensity cases). Because of the second term in Eq. (1), this change in resistivity gradient drives a magnetic field reversal [see Fig. 4(c)], resulting in a perturbation in the beam current density, producing a localized increase near the edge of the


FIG. 4 (color online). Hybrid PIC simulation results using the QMD Kubo-Greenwood model (outputs all at an example simulation time equal to $1.4 \mathrm{ps}$ after the start of the laser pulse). (a) $\log _{10} 2 \mathrm{D}$ target temperature map (in eV) with selected isothermal contours for the $5 \times 10^{20} \mathrm{~W} \mathrm{~cm}^{-2}$ case. Transverse (b) temperature and (d) resistivity profiles at $50 \mu \mathrm{m}$ depth for $5 \times 10^{19}$ and $5 \times 10^{20} \mathrm{~W} \mathrm{~cm}^{-2}$. Dashed vertical lines for both laser intensity cases illustrate that a dip in the resistivity profile, which seeds beam hollowing, arises at a temperature of about $3.5 \mathrm{eV}$. (c) $2 \mathrm{D}$ map of magnetic flux density $\left(B_{Z}\right.$ component in Tesla) for the $5 \times 10^{20} \mathrm{~W} \mathrm{~cm}^{-2}$ case, showing a reversal in magnetic field direction inside the edge of the beam.

beam. Subsequently, the resulting increased return current, and therefore heating in this region, drives a localized increase in resistivity for temperatures above $\sim 3.5 \mathrm{eV}$ (up to tens of eV-see Fig. 1) and the region near the edge of the beam remains more resistive than the center [see the resistivity spikes in Fig. 4(d), which grow as the simulation evolves]. This, together with the pinching effect of the magnetic field arising from the first term in Eq. (1), due to the higher current density, leads to strong positive feedback which sustains the annular transport pattern as the beam propagates through the remainder of the target. Figure 4(c) shows a 2D map of the magnetic field, illustrating the strong reversal in magnetic field just inside the beam edge.

For the lower intensity case, although a small reversal in resistivity [Fig. 4(d)] and therefore magnetic field direction is induced at the beam edge, the resulting current density perturbation is not large enough to seed an annular transport pattern.

This study demonstrates, for the first time, that even subtle features in the low-temperature, few-eV region of the resistivity-temperature curve can profoundly alter the fast electron transport pattern in solids. It also provides new understanding of the origin of annular transport patterns [26-28] and highlights the potential to induce and control annular beams for applications (e.g., for fast-ignition ICF). 
This could be achieved, for example, by engineering a target resistivity-temperature profile by doping and by variation of laser drive parameters to create a desired resistivity evolution in space and time.

We acknowledge the expert support of the staff at the Central Laser Facility of the Rutherford Appleton Laboratory and the use of computing resources provided by the STFC e-Science Facility. This work is supported by EPSRC (Grant No. EP/J003832/1), the Swedish Research Council, and by the National Science Foundation of China (Grant No. 11205100).

*paul.mckenna@strath.ac.uk

[1] M. Tabak, J. Hammer, M.E. Glinsky, W. L. Kruer, S. C. Wilks, J. Woodworth, E. M. Campbell, M. D. Perry, and R. J. Mason, Phys. Plasmas 1, 1626 (1994).

[2] M. Borghesi, J. Fuchs, S. V. Bulanov, A. J. Mackinnon, P. K. Patel, and M. Roth, Fusion Sci. Technol. 49, 412 (2006).

[3] S. Kar, A. P. L. Robinson, D. C. Carroll, O. Lundh, K. Markey, P. McKenna, P. Norreys, and M. Zepf, Phys. Rev. Lett. 102, 055001 (2009).

[4] B. Ramakrishna et al., Phys. Rev. Lett. 105, 135001 (2010).

[5] R. H. H. Scott et al., Phys. Rev. Lett. 109, 015001 (2012).

[6] J. R. Davies, Phys. Rev. E 69, 065402 (2004).

[7] M. Temporal, J. J. Honrubia, and S. Atzeni, Phys. Plasmas 15, 052702 (2008).

[8] A. R. Bell, J. R. Davies, S. Guerin, and H. Ruhl, Plasma Phys. Controlled Fusion 39, 653 (1997).
[9] L. Gremillet, G. Bonnaud, and F. Amiranoff, Phys. Plasmas 9, 941 (2002).

[10] A. R. Bell and R. J. Kingham, Phys. Rev. Lett. 91, 035003 (2003).

[11] L. Spitzer and R. Harm, Phys. Rev. 89, 977 (1953).

[12] P. McKenna et al., Phys. Rev. Lett. 106, 185004 (2011).

[13] Y. T. Lee and R.M. More, Phys. Fluids 27, 1273 (1984).

[14] P. L. Silvestrelli, A. Alavi, M. Parrinello, and D. Frenkel, Phys. Rev. Lett. 77, 3149 (1996).

[15] M. P. Desjarlais, J. D. Kress, and L. A. Collins, Phys. Rev. E 66, 025401 (2002).

[16] G. Kresse and J. Hafner, Phys. Rev. B 47, 558 (1993).

[17] A. P. L. Robinson, M. H. Key, and M. Tabak, Phys. Rev. Lett. 108, 125004 (2012).

[18] J. R. Davies, A. R. Bell, M. G. Haines, and S. M. Guerin, Phys. Rev. E 56, 7193 (1997).

[19] J. R. Davies, Phys. Rev. E 65, 026407 (2002).

[20] S. C. Wilks and W. L. Kruer, IEEE J. Quantum Electron. 33, 1954 (1997).

[21] M. Coury et al., Phys. Plasmas 20, 043104 (2013).

[22] X. H. Yuan et al., New J. Phys. 12, 063018 (2010).

[23] J. Fuchs et al., Phys. Rev. Lett. 91, 255002 (2003).

[24] M. N. Quinn et al., Plasma Phys. Controlled Fusion 53, 124012 (2011).

[25] J. R. Davies, J. S. Green, and P. A. Norreys, Plasma Phys. Controlled Fusion 48, 1181 (2006).

[26] M. Storm et al., Phys. Rev. Lett. 102, 235004 (2009).

[27] P. A. Norreys et al., Plasma Phys. Controlled Fusion 48, L11 (2006).

[28] J. A. Koch, M. H. Key, R. R. Freeman, S. P. Hatchett, R. W. Lee, D. Pennington, R. B. Stephens, and M. Tabak, Phys. Rev. E 65, 016410 (2001). 